\title{
PREPARATION, CHARACTERIZATION AND ANTI-CANCER ACTIVITY OF NANOSTRUCTURED LIPID CARRIERS CONTAINING IMATINIB
}

Hafiz A. Makeen*¹, Syam Mohan², Mohamed Ahmed Al-Kasim³, Muhammad Hadi Sultan ${ }^{4}$, Ahmed A Albarraq ${ }^{1}$, Rayan A. Ahmed ${ }^{3}$, Hassan A. Alhazmi ${ }^{2}$, M. Intakhab Alam*4

${ }^{1}$ Department of Clinical Pharmacy, College of Pharmacy, Jazan University, Jazan, KSA

${ }^{2}$ Substance Abuse and Toxicology Research Centre, Jazan University, Jazan, KSA

${ }^{3}$ Department of Pharmacology, College of Pharmacy, Jazan University, Jazan, KSA

${ }^{4}$ Department of Pharmaceutics, College of Pharmacy, Jazan University, Jazan, KSA

*Corresponding authors: Hafiz A. Makeen

Department of Clinical Pharmacy, College of Pharmacy, Jazan University, Jazan, KSA

Tel: +96673342662; E-mail: hafiz@jazanu.edu.sa

M. Intakhab Alam

Department of Pharmaceutics, College of Pharmacy, Jazan University, Jazan, KSA

Tel: +966531438951; E-mail: $\underline{\text { mialam@jazanu.edu.sa }}$ 


\begin{abstract}
Breast cancer is the most widespread malignancy in women from corner to corner of the world. Nanostructured lipid carrier (NLC) proved its efficacy in the treatment of cancer. NLC loaded with Imatinib (IMA) (NANIMA) was prepared and evaluated for in vitro efficacy in MCF-7 breast cancer cells. Hot homogenization method was used for the preparation of NANIMAs. Aqueous solution of surfactants (hot) was mixed with molten mixture of stearic acid and sesame oil (hot) under homogenization. The prepared NANIMAs were characterized and evaluated for size, polydispersity index, zeta potential, encapsulation efficiency, release studies, stability studies and MTT assay (cytotoxicity studies). The optimized NANIMA revealed particle size of $104.63 \pm 9.55$ d.nm, PdI of $0.227 \pm 0.06$ and EE of $99.79 \pm 0.03$. All NANIMAs revealed slow and sustained release behaviour. The surfactants used in the preparation of NANIMAs exhibited their effects on particle size, zeta potential, encapsulation efficiency, stability studies and release studies. The cytotoxicity studies unveiled 8.75 times increase in cytotoxicity for optimized NANIMA $\left(\mathrm{IC}_{50}=6\right.$ $\mu \mathrm{M})$ when compared with IMA alone $\left(\mathrm{IC}_{50}=52.5 \mu \mathrm{M}\right)$ on MCF-7 breast cancer cells. After this, NLC containing IMA possibly will be employed for the cure of breast cancer. A lesser amount of IMA loaded NLC will be sufficient than IMA alone for the treatment of breast cancer. Moreover, the patient will exhibit not as much of adverse effects as in case of IMA alone. Consequently, NANIMA could prove to be useful for effective therapeutic outcome in breast cancer treatment.
\end{abstract}

Key words: Imatinib, NLC, Tween 80, SLS, MTT Assay, Release study, Drug delivery, Breast cancer. 


\section{Introduction}

Breast cancer is the most prevalent malignancy in women across the world and is curable in approximately $80 \%$ of patients with early-stage non-metastatic disease. With treatments presently available, advanced breast cancer with remote organ metastases is considered incurable [1]. On the molecular level, its progression is involved with regulation of many pathways. Receptor tyrosine kinase over-expression is a typical pathway correlated with different malignancies [2]. At Novartis, Imatinib has been synthesized and has emerged as the lead compound against chronic myelogenous leukemia (CML) cells for clinical development [3]. Centered on its application in the treatment of CML and gastrointestinal stromal tumors (GISTs), imatinib is a tyrosine kinase inhibitor that usually inhibits the tyrosine kinase action of BCR-Abl, platelet-derived growth factor receptors (PDGFRs), and KIT4 proteins. Tyrosine kinase receptors that play an essential role in tumour development, pathologic angiogenesis, and metastatic progression of breast cancer via the phosphorylation of target proteins [4]. It has been established that the inhibition of the function of the receptor tyrosine kinase involved in the EGFR signaling cascade forms the foundation for the usage of EGFR specific inhibitors of tyrosine kinase, exemplified by gefitinib kinase [5]. Thus, the structurally similar drug imatinib also expected to perform with breast cancer inhibition. But, in cancer therapy, the therapeutic potency of imatinib mesylate is impaired by its off-target cardiotoxicity [6].

Lipid nanocarriers revealed a great potential for delivery of numerous anti-cancer agents. It was seen to have benefits over traditional chemotherapy (e.g., drug resistance and adverse effect). Furthermore, the benefits associated with the particular properties of lipid nanocarriers include tumor-specific drug deposition, efficient pharmacokinetics and pharmacodynamics account of therapeutic agent, enhanced internalization and intracellular transport $\quad, \quad$ and decreased biodistribution, resulting in the mitigation of harmful consequences of anti-tumor treatments $[7,8]$. The 
administration of various drug molecules such as gefitinib [9], celecoxib [10], doxorubicin [11], duloxetine [12], tamoxifen [13], curcumin [14], and paclitaxel [15] has been extensively investigated using nanostructured lipid carriers (NLC). Because of their fast intake by cells, biodegradability, and bio-acceptability [9], NLCs are being studied extensively for drug delivery. It has better controlled release features and drug loading capability, as well as a lower burst release rate [16]. Furthermore, the use of biocompatible lipids and the involvement of a metabolic pathway in living systems decrease the possibility of toxicity [17]. NLC is made up of two kinds of lipids: solid lipid (fat) and liquid lipid (liquid) (oil). The inclusion of liquid lipid (oil) improves the drug delivery properties of NLC as compared to solid lipid nanoparticles (SLN) [18]. As a result, NLCs are claimed to be a new generation of SLN that integrates the qualities of SLN while still overcoming the drawbacks.

The aim of this research was to design and formulate NLC loaded IMA (NANIMA), then evaluate in vitro characteristics such as particle size, polydispersity index, zeta potential, encapsulation performance, release performance, stability, and cytotoxicity (MTT assay) studies in MCF-7 breast cancer cells in order to minimize the therapeutic dose of IMA.

\section{Materials and Methods}

Imatinib was purchased from LC Laboratories (Woburn, MA, USA). Stearic acid (SA) and sodium lauryl sulphate (SLS) were obtained from Himedia (Mumbai, India). Sesame oil (pure) was obtained from the local merchant (Jazan, Saudi Arabia). Tween 80 (T80) was obtained from Loba Chemei (Mumbai, India). RPMI-1640 medium, FBS and penicillin-streptomycin were purchased from Gibco (Invitrogen Corp., USA). Neutral red was purchased from Sigma Chemical Co. (St Louis, MO, USA). All other chemicals and reagents used were of analytical grade.

\section{Preparation of NANIMA}


IMA loaded NLC (NANIMA) was prepared by the method of homogenization (hot). The stearic acid (fat) and sesame oil were used as lipid ingredients in the preparation of NANIMA formulations. The hot aqueous solution of surfactants in a beaker was transferred to another beaker containing molten mixture of lipids and mixed with the help of a homogenizer (HG-15D, WiseTis, Germany). The mixing was done for a period of $20 \mathrm{~min}$ at a speed of $5000 \mathrm{rpm}$. The aqueous solution of surfactants was prepared by dissolving sodium lauryl sulphate (SLS) and tween 80 (T80) in $25 \mathrm{ml}$ of purified water (Table 1). The lipid phase was consisted of stearic acid (SA) (0.5 g), sesame oil (0.25 ml) and IMA (0.05 g).

Table 1: Amount of surfactants used in the preparation of NANIMA formulations

\begin{tabular}{|l|c|c|}
\hline Formulation & Sodium lauryl sulphate (SLS) $(\mathbf{m g})$ & Tween 80 $($ T80) $(\boldsymbol{\mu l})$ \\
\hline STIN & 50 & 25 \\
\hline S2TIN & 75 & 25 \\
\hline TSIN & 25 & 75 \\
\hline T2SIN & 25 & 100 \\
\hline
\end{tabular}

\section{Particle size, polydispersity index and zeta potential}

The photon correlation spectroscopy (Malvern, Nano ZS90, UK) was used for the assessment of characterization parameters including particle size (as mean), polydispersity index (PdI) and zeta potential. The study was performed after proper dilution of formulations with Millipore water. The appropriate concentration of particles was obtained after dilution to circumvent multi-scattering actions. All measurements were made in triplicate.

Encapsulation efficiency (EE) 
The encapsulation efficiency (EE) was determined in terms of percentage quantity of IMA trapped in NLC. The ultrafiltration centrifugation method was followed for the estimation of EE (\%) of all NANIMAs. The method consists of centrifugation of ultra-filter tube (3K, Millipore Ireland) containing $2 \mathrm{ml}(\approx 4 \mathrm{mg}$ of IMA) of the formulation at a speed of $4000 \mathrm{~g}$ for a period of $20 \mathrm{~min}$. The free IMA was estimated in the filtrate obtained after centrifugation. The filtrate was analyzed spectrophotometrically after proper dilution. The spectrophotometric analysis was carried out at $265 \mathrm{~nm}$ by means of a spectrophotometer (UV, Shimadzu, Kyoto, Japan). The EE was estimated using the formula mentioned below:

$$
\mathrm{EE}(\%)=\frac{\text { Quantity of IMA taken }- \text { Quantity of IMA obtained }}{\text { Quantity of IMA taken }} \times 100
$$

\section{Lyophilization}

The aqueous solution of mannitol $(6 \% \mathrm{w} / \mathrm{v})$ was prepared and used as cryoprotectant in freeze drying on NANIMA formulations. The aqueous dispersion of NANIMAs was mixed with mannitol solution (1:4) followed by freezing in an ultra-low temperature freezer (U9280-0002, New Brunswick, Germany) at $-70^{\circ} \mathrm{C}$ for $12 \mathrm{~h}$. The frozen samples were lyophilized using a lab freeze dryer (BT85, Millrock technology, Kingston, NY). After 24 h, dried samples were collected and stored in suitable containers.

\section{Scanning electron microscopy (SEM)}

Scanning electron microscope (Zeiss EVO LS10; United Kingdom) was used for taking the micrographs of NANIMAs. The both side adhesive carbon tape (SPI Supplies, West Chester, USA) was used for fixing the NANIMA samples. Moreover, the coating was done in an argon atmosphere at $20 \mathrm{~mA}$ for 60 seconds under vacuum with gold in a Q150R sputter coater unit (Quorum Technologies Ltd. East Sussex, UK).

\section{In vitro release study}


The in vitro release study was performed for all NANIMA formulations. The dialysis bag (12-14 KD MWCO) (Spectrum Laboratories, Inc. CA, USA) method was used to perform the study. The formulation ( $1 \mathrm{ml}$; equivalent to $2 \mathrm{mg}$ of IMA) was transferred to the dialysis bag. It was followed by fastening both ends of the bag with thread. The bag was placed in a beaker containing phosphate buffer solution $(\mathrm{pH} 7.4)(300 \mathrm{ml})$ mixed with tween $20(1 \% \mathrm{v} / \mathrm{v})$ as release media. The stirring of media was performed using a bead on a magnetic stirrer at a speed of $50 \mathrm{rpm}$ and at a temperature of $37^{\circ} \mathrm{C}$. An aliquot $(2 \mathrm{ml})$ was taken from the beaker after $0.5,1,2,3,4,5,6,12,24 \mathrm{~h}$ followed by replacement by fresh media. The collected sample was passed through a syringe fitted with filter $(0.45 \mu \mathrm{m})$ and filtered sample was obtained. It was followed by analysis for drug content by UVspectrophotometer (Schimadzu, Japan) at a $\lambda_{\max }$ of $265 \mathrm{~nm}$.

\section{Stability studies}

The NANIMA formulations were subjected to stability studies and analyzed under the influence of definite temperature. The NANIMA formulations were stored at room temperature $\left(25^{\circ} \mathrm{C}\right)$ for a period of three months. These were then, analyzed for different physical changes including consistency, color, aggregation and smell. Moreover, the characterization parameters including size, zeta potential, polydispersity index and encapsulation efficiency were used to evaluate the influence of temperature.

\section{Cell line and cell cultures}

The current study was conducted using MCF-7 cells (ER-positive breast cancer cell) which were purchased from ATCC. The cells were grown in $\mathrm{CO}_{2}$ incubator (New Brunswick Scientific) at $37^{\circ} \mathrm{C}$ in using RPMI-1640 media which was supplemented with $10 \%$ Fetal bovine serum and penicillin $(100 \mathrm{U} / \mathrm{ml})$, streptomycin $(100 \mathrm{~g} / \mathrm{ml})$. The samples were diluted to desired concentration using serum free media and treated for the required time period.

\section{Cell viability assay}


The cytotoxicity assay was carried out by colorimetric MTT viability assay [19]. Briefly, different sample concentrations were put in triplicates and incubated for $72 \mathrm{~h}$. Each plate were included with untreated cell controls and blank cell-free control. MTT $(5 \mathrm{mg} / \mathrm{ml})$ was provided to each well after incubation and the plates were further incubated for an additional $4 \mathrm{~h}$, after which the media was removed. To solubilize the formazan crystals, DMSO $(100 \mu \mathrm{l})$ was inserted into each well. The absorbance was read using a microtiter plate reader at a wavelength of $490 \mathrm{~nm}$ (BioTek Instruments, Winooski, VT, USA). With the necessary controls taken into account, the percentage of cellular viability was determined. In triplicate, the experiment was completed. The cell proliferation inhibitory rate was estimated using the following formula:

$$
\text { Growth inhibition }=\frac{\text { OD control }- \text { OD treated }}{\text { OD control }} \times 100
$$

The cytotoxicity of our formulations on cancer cells was expressed as $\mathrm{IC}_{50}$ values (the sample concentration reducing the cell count of treated cells by $50 \%$ with respect to untreated cells).

\section{Detection of Apoptosis by acridine orange (AO) propidium iodide (PI) double staining}

IMA and S2TIN induced cell death in MCF-7 breast cancer cells was quantified using AO and PI double-staining according to standard procedures and examined under a fluorescence microscope. Briefly, treatment was carried out in T-flasks. Cells were plated at a concentration of $1 \times 10^{6}$ cells/ml for $24 \mathrm{~h}$, the media was substituted with new media containing IMA $52.5 \mu \mathrm{M}$ and S2TIN 6 $\mu \mathrm{M}$ along with a blank control. The cells were incubated for $72 \mathrm{~h}$. Floating and adhering cells were collected, centrifuged at $300 \times \mathrm{g}$ for $10 \mathrm{~min}$, washed twice with PBS to remove the remaining media. The supernatant was discarded, and the cells were stained with fluorescent dyes containing AO $(10 \mathrm{mg} / \mathrm{ml})$ and PI $(10 \mathrm{mg} / \mathrm{ml})$. The freshly stained cell suspension was dropped onto a glass slide and covered with a coverslip. Slides were observed under the ultraviolet-fluorescence microscope within $30 \mathrm{~min}$ before the fluorescent color starts to fade. The viable, early-dependent 
phospholipid-binding protein, the plasma membrane alterations, apoptotic, and late apoptotic cells were observed.

\section{Statistical analysis}

All data is reported as means standard deviation $( \pm \mathrm{SD})$. For statistical analysis, one-way ANOVA was used, and statistically significant differences were described as p-values less than 0.05 (p < $0.05)$.

\section{Result and Discussion}

NANIMA was prepared using the fat and oil by hot homogenization method [9]. Moreover, the surfactants including SLS and T80 were used for the stabilization of the system. The NANIMA formulations were evaluated for particle size, zeta potential, SEM, release studies, stability studies and cytotoxicity studies.

\section{Particle size, polydispersity index and zeta potential}

NANIMAs were evaluated with the purpose of determination of their particle size distribution, zeta potential and PdI values using Malvern Zetasizer. The mean particle size was observed to be 92.6, 104.63, 111.15 and $102.57 \mathrm{~nm}$ for STIN, S2TIN, TSIN and T2SIN respectively (Table 2). The influence of surfactants (type and amount) on particle size of NANIMAs was analyzed. The size of NANIMAs was observed to be more (S2TIN>STIN) when surfactant (SLS) concentration was increased. However, contrasting result was observed in case of T80 (size decreased after increasing T80 concentration) (TSIN>T2SIN). However, the result was found to be non-significant. Thus, the particle size exhibited insignificant effect due to variation in amount of surfactants (T80 and SLS) in NLC containing IMA.

Particle size is considered to be a fundamental factor in the development of nano-therapeutic systems. The composition of formulation including surfactant, lipids and drug greatly affect the 
particle size. Moreover, the process parameters such as speed and duration of homogenization, and temperature positively affect the size of NLCs.

Particle size exerts its effect not only on stability but also significantly affects the cellular uptake by tumor cells and passive targeting of anti-cancer agents to tumours. ${ }^{20}$ Chemotherapeutic agents to be delivered effectively the particle size are considered to be in the range between $50-200 \mathrm{~nm}$. The appropriateness of nanocarriers between 50 and $100 \mathrm{~nm}$ for avoiding monocellular phagocytic system uptake and prolonging blood circulation time is investigated. Nanocarriers smaller than 200 $\mathrm{nm}$ have been found to be effective at passive targeting to tumor tissues through enhanced permeability and retention (EPR) [21].

The polydispersity index (PdI) of NANIMAs was observed to be $0.301,0.227,0.313$ and 0.312 for STIN, S2TIN, TSIN and T2SIN respectively (Table 2). PdI is reported to be a measure of aggregation/agglomeration of particles in nanosystems. Thus, the mono-disperse conduct of the system is predicted when PdI value is closer to zero. Moreover, the polydispersity of the system is expected with the higher value of PdI $(>0.5)$. The monodispersed systems have lower agglomeration propensity than the polydispersed systems [22]. The physical stability of colloidal dispersions can be predicted by zeta potential (ZP) values. It is known as a primary predictor of nanocarrier stability, including NANIMA preparations. Furthermore, a certain value of ZP of nanocarriers improves their ability to bind to cell membrane. For the transport of therapeutic agents, a particular value of zeta potential is needed to produce an electrostatic contact with cell membranes [23]. The higher possibility of stabilization of colloidal systems can be achieved by the higher values of ZP irrespective of type of charge (positive or negative). Characteristically, stability (no aggregation/flocculation) of the system is achieved when charged particles with ZP value of $(>|20|)$ are present [24]. Thus, this much of $\mathrm{ZP}$ is found to be appropriate to retain the electrostatic repulsion among the similarly charged particles for the prevention of aggregation. ZP 
of NANIMA is characterized as the potential contrast between NLC surface and its fluid medium (i.e., particle - liquid interface). The charge of all NANIMAs was found to be negative (negative ZP). The negative charges of NANIMAs may be contributed by slightly ionized fatty acids [25]. The ZP of NANIMAs increased when increasing the quantity of T80. Highest ZP (negative) was observed for T2SIN containing highest amount of T80 among all NANIMAs. Thus, T2SIN is assumed to be most stable electrostatically among all NANIMAs based on the value of ZP.

The lower ZP value can be explained by the shielding of electrostatic charges on NANIMAs by non-specifically adsorbed non-ionic surfactants (T80) [26]. Furthermore, the higher ZP value can be explained by the availability of adsorption space on the surface of NANIMAs particles. Subsequently, the sequence of stable formulations of NANIMA can be arranged as T2SIN > S2TIN $>$ TSIN $>$ STIN.

\section{Encapsulation efficiency (EE)}

The estimation of EE is employed to differentiate the amount of IMA that was incorporated into NLC from the free IMA. The NANIMA formulations were found to exhibit high EE value (from $98.50 \pm 2.43 \%$ to $99.79 \pm 0.03$ ) (Table 2 ). The solubility of IMA in lipid phase can account for high value of EE. Moreover, the high EE value may also be contributed by the partitioning of IMA between aqueous phase and oil phase.

The accommodation of more quantities of IMA in NANIMA (thus high value of EE) may also be explained on the basis of introduction of sesame oil into stearic acid, which increases the imperfections in the crystal lattice and decreases crystalline order. It has been investigated that the lower degree of crystalline order in lipid nanocarriers is considered to be responsible for the increase in capability to accommodate more drugs [27]. Hence, amid the fatty acid chains and/or lipid layers the IMA molecules can be accommodated in higher amount. Moreover, the parallel outcomes were perceived previously [9, 28, 29, 30, 31]. 
The high value of EE may also be attributed to the concentration of surfactants. The increase in viscosity of aqueous phase and addition of surfactants may result in high value of $\mathrm{EE}$ after decreasing the diffusion speed of IMA. The smaller size of particles give increased surface area of NANIMA where more IMA molecules were incorporated. Thus, the availability of adequate surfactant enables IMA to remain entrapped within the lipid and consequently lead to high value of EE. Furthermore, the micelle forming surfactants including SLS form monomers and micelles on the lipid surface that give alternative sites for IMA incorporation [32]. The effect of concentrations of surfactant on the values of EE were found to be in compliance with the outcomes of others [28, $30,33]$.

Table 2: Characterization (size, polydispersity index - PdI, zeta potential, encapsulation efficiency - EE) outcomes of NANIMAs

\begin{tabular}{|c|c|c|c|c|}
\hline $\begin{array}{l}\text { Formulation } \\
\text { Code }\end{array}$ & $\begin{array}{l}\text { Size (d.nm) } \\
(n=3)( \pm S D)\end{array}$ & $\begin{array}{l}\text { PdI }(n=3) \\
( \pm \text { SD })\end{array}$ & $\begin{array}{l}\text { Zeta Potential } \\
(\mathbf{m V})(\mathbf{n}=\mathbf{3})( \pm \mathrm{SD})\end{array}$ & $\begin{array}{l}\text { EE }(\%)(\mathrm{n}=3) \\
( \pm \mathrm{SD})\end{array}$ \\
\hline STIN & $92.60 \pm 23.47$ & $0.301 \pm 0.07$ & $24.83 \pm 2.90$ & $98.50 \pm 2.43$ \\
\hline S2TIN & $104.63 \pm 9.55$ & $0.227 \pm 0.06$ & $27.70 \pm 9.30$ & $99.79 \pm 0.03$ \\
\hline TSIN & $111.15 \pm 19.91$ & $0.313 \pm 0.07$ & $26.40 \pm 5.09$ & $99.63 \pm 0.53$ \\
\hline T2SIN & $102.570 \pm 12.26$ & $0.312 \pm 0.08$ & $29.57 \pm 3.94$ & $99.66 \pm 0.13$ \\
\hline
\end{tabular}

\section{Scanning electron microscopy (SEM)}

SEM analysis was accomplished to acquire the information about the morphology (shape together with size) of NANIMAs. Two types of samples were used for SEM analysis of NANIMAs. First type was lyophilized samples. Second type sample was prepared by dispersing of lyophilized NANIMAs in water and drop casted on a glass slide. The morphology and size of all NANIMAs were found to be in nanometer range. However, the lyophilized NANIMAs revealed elongated and 
parallelepiped shape (Figure 1). Thus, NANIMAs with mannitol as cryoprotectant seemed to adopt orthorhombic form with flattened structures.
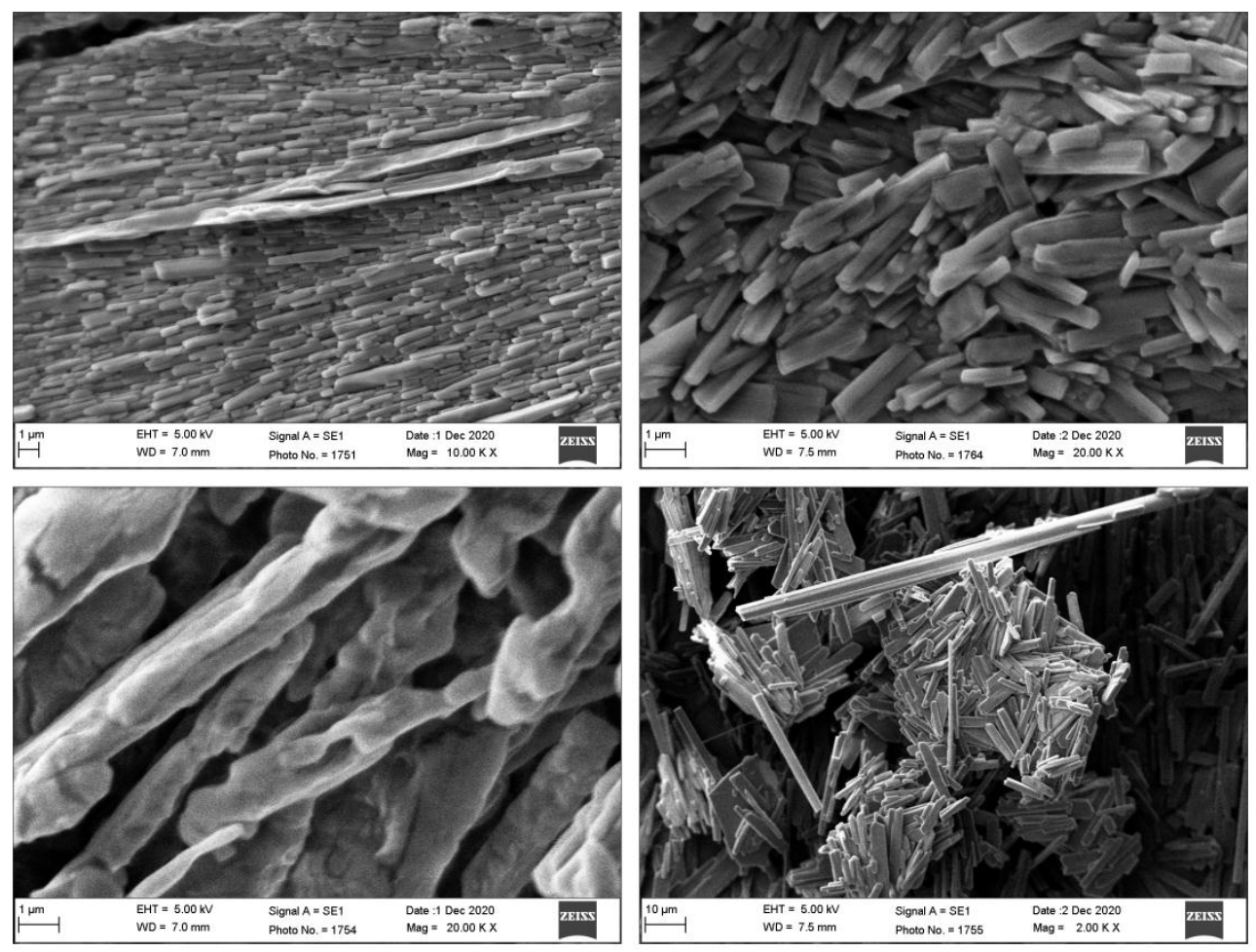

Figure 1: SEM images of lyophilized NANIMAs including STIN (upper left), S2TIN (upper right), TSIN (lower left) and T2SIN (lower right).

Moreover, it revealed agglomeration of particles which might be either due to the lipid nature, concentration of dispersion medium or shrinkage of NLCs during drying of NANIMAs. The SEM images obtained after reconstitution revealed spherical shape and more or less smooth surface (Figure 2). 

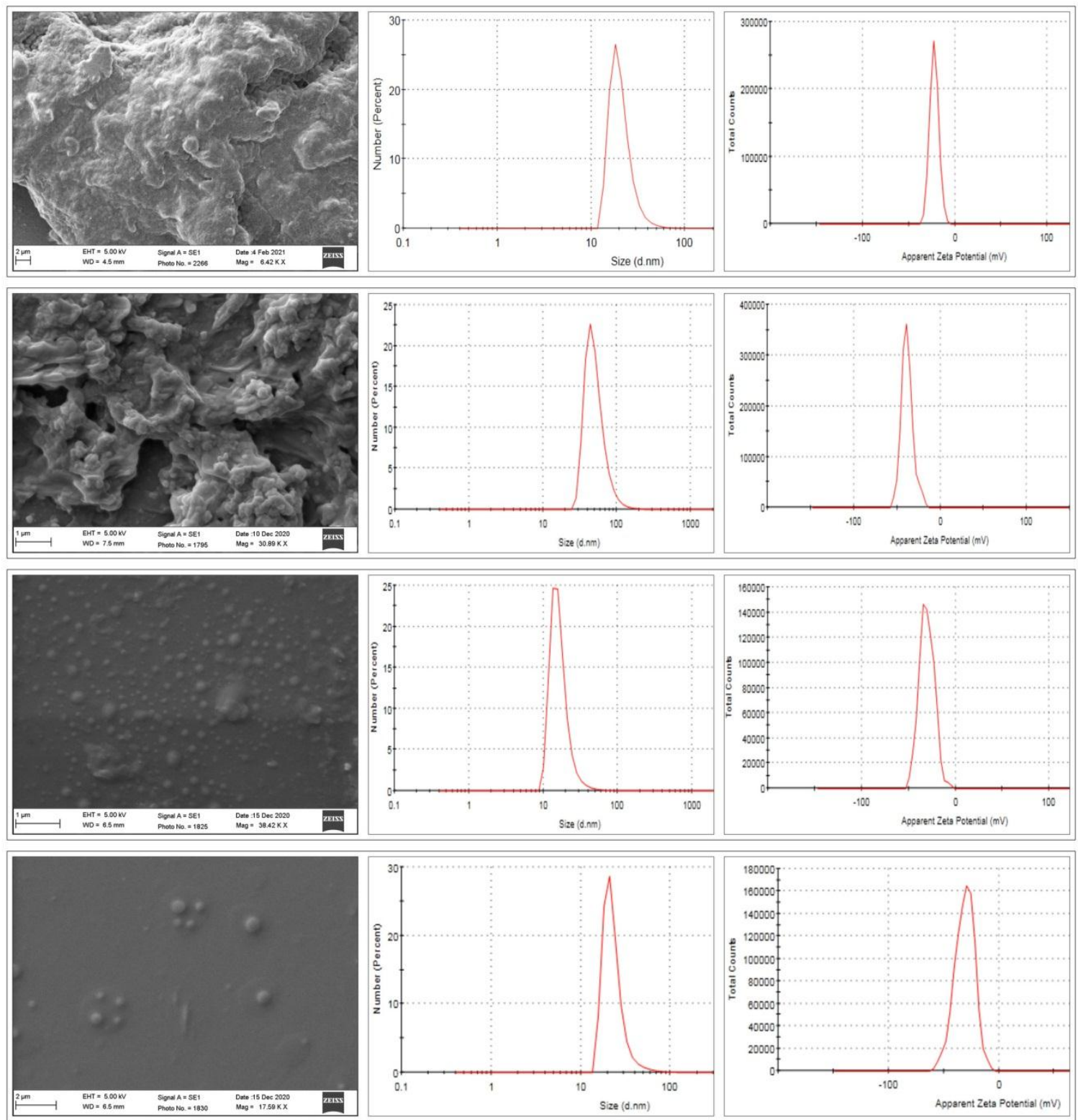

Figure 2: SEM (obtained by second method), size and zeta potential of STIN (first from top) ( d.nm, ZP = -38.2 mV); TSIN (third from top) (size = 88.65 d.nm, ZP = -30.5 mV); T2SIN (lower most) $($ size $=91.11 \mathrm{~d} . \mathrm{nm}, \mathrm{ZP}=-34.1 \mathrm{mV})(\mathrm{n}=1)$.

\section{In vitro release study}

Figure 3 depicts the release characteristics of NANIMA formulations. The in vitro release tests of NANIMA formulations were carried out using the dialysis bag diffusion technique. To avoid 
interference from IMA solubility in the release medium, the sink conditions were preserved throughout the study. The slow and sustained release of IMA was exhibited by all NANIMA formulations, as expected for NLC formulations. The release of IMA from within the NLC core, as well as partitioning between the water and lipid matrix, may be attributing factors for lipid nanocarriers like NLC's long-term release behavior [34]. It could also be attributed to the interfacial membrane's barrier function, the solid matrix of NLC, and the subsequent immobilization [24]. The slow release of IMA from NLCs could be explained by entrapment homogeneity throughout the system [25]. Furthermore, the release characteristics of NANIMA formulations were supported the high value of $\mathrm{EE}(>99 \%)$. Consequently, the prolonged anti-cancer action may be obtained from a single dose NLC containing anti-cancer agent. Thus the suitability of NLC may be considered for the delivery of IMA.

The release studies exhibited absence of burst effect that can be observed in the release pattern shown in Figure 3. It may be attributed to the high value of $\mathrm{EE}$ and negligible amount of unentrapped IMA. Generally, the presence of unentrapped drug on the surface of NLC may contribute to the burst effect in release studies [29, 35]. As shown in Table 2, all NANIMA formulations were found to have EE value of nearly hundred percent (> 99\%), which is supported by release tests.

The IMA solution was prepared by dissolving in ethanol and aqueous media was added. The release of IMA from IMA solution was found to be faster than NANIMA formulations. It may be because of sustaining the release of IMA by dialysis membrane only.

However, the NANIMA formulations revealed sustained release performance that may be credited to both NLC as well as dialysis membrane. S2TIN revealed the highest and TSIN exhibited the least amount of IMA released after $24 \mathrm{~h}$ of study among all the NANIMA formulations. STIN and T2SIN exhibited nearly equal released amount of IMA after $24 \mathrm{~h}$. 
Thus, different factors may be considered responsible for affecting the release results of IMA from NANIMA formulations including nature and amount of surfactants and duration of sampling. SLS appears to be extra influential than T80 on the release of IMA from NANIMA formulations (S2TIN > T2SIN and S2TIN > TSIN). Moreover, the higher amount of SLS gives higher released amount of IMA (S2TIN > STIN). However, in case of T80, even higher amount (T2SIN) seems to be ineffective to mobilize IMA excellently from the solid core of NLC to be exhibited as the released amount more than the lesser amount (S2TIN) of SLS. Reliably, the collective impact of SLS and T80 on the release behaviour of IMA was more recognizable in S2TIN than STIN, TSIN and T2SIN. It may be explained on the basis of sampling gap and combined effect of surfactants. A sampling gap of $12 \mathrm{~h}$ provided ample duration of time for the action of surfactants and accretion of released IMA may be considered a dependable factor for more release.

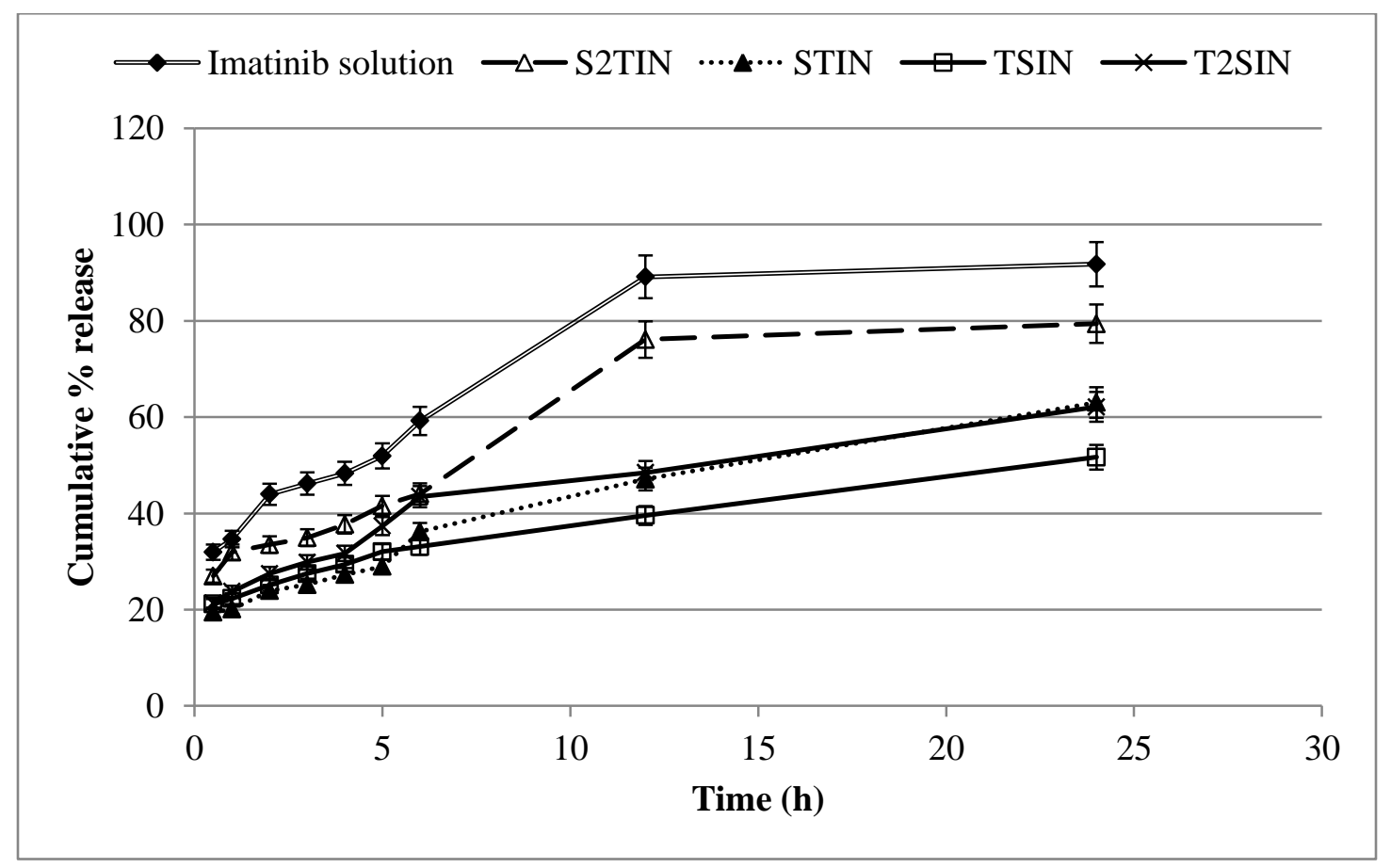

Figure 3: In vitro release pattern of NANIMA formulations

Moreover, the NANIMA formulations were evaluated for the mechanism of release of IMA. The release data of NANIMAs were fitted into different kinetic models (Figure 4). The preferred model 
that fits best to the release data was chosen on the premise of the higher value of correlation coefficients $\left(\mathrm{R}^{2}\right)$. Accordingly, Higuchi model (diffusion controlled profile) was followed all NANIMA formulations including STIN $\left(\mathrm{R}^{2}=0.974\right)$, S2TIN $\left(\mathrm{R}^{2}=0.904\right)$, TSIN $\left(\mathrm{R}^{2}=0.996\right)$, T2SIN $\left(\mathrm{R}^{2}=0.975\right)$. Consequently, all NANIMA formulations exhibited release of IMA by diffusion controlled mechanism based on the Fick's law which is square root time dependent.

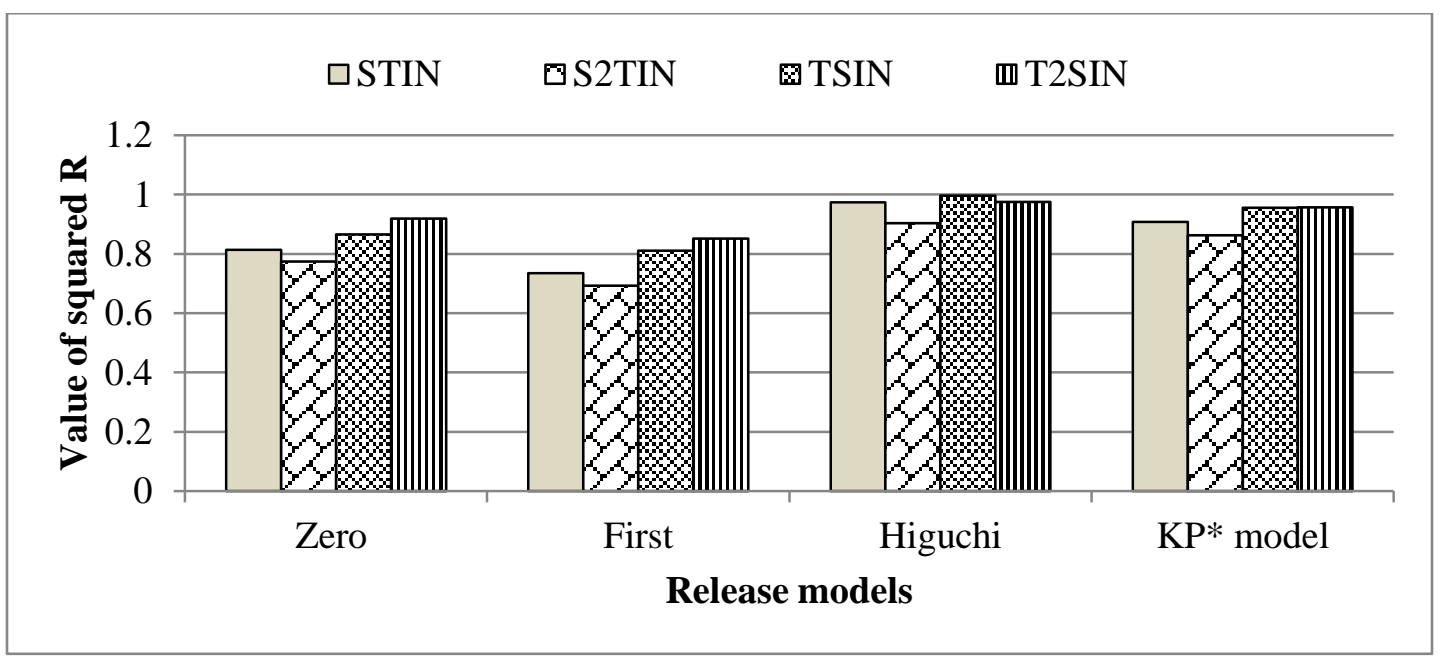

Figure 4: Correlation coefficients (R2) value for IMA release from different NANIMA formulations (*Korsmeyer-Peppas)

\section{Stability studies}

Among the factors affecting the stability of lipid nanocarriers including NLCs, temperature is considered to be a significant one. Storage at room temperature $\left(25^{\circ} \mathrm{C}\right)$ does not require any special condition. Thus, stability at room temperature $\left(25^{\circ} \mathrm{C}\right)$ is considered to be commendable. Accordingly, the NANIMA formulations exhibited temperature effect on particle size, polydispersity index (PdI), encapsulation efficiency (EE \%) and zeta potential (ZP) after three months of storage. The outcome of temperature effects was observed as shown in Figure 5, 6, 7 \& 8. The effect was observed in the favour of particle growth in case of TSIN followed by T2SIN, S2TIN and STIN (i.e., TSIN > T2SIN > S2TIN > STIN). However, the particle size of NANIMA 
formulations remained in colloidal nanometer range $(<550 \mathrm{~nm})[36]$. Since, the increase in size of TSIN was not more than two folds, so it indicates the nonappearance of aggregation. This kind of variations may possibly be ascribed to the swelling or adsorption of extra surfactants on particle surface [18].

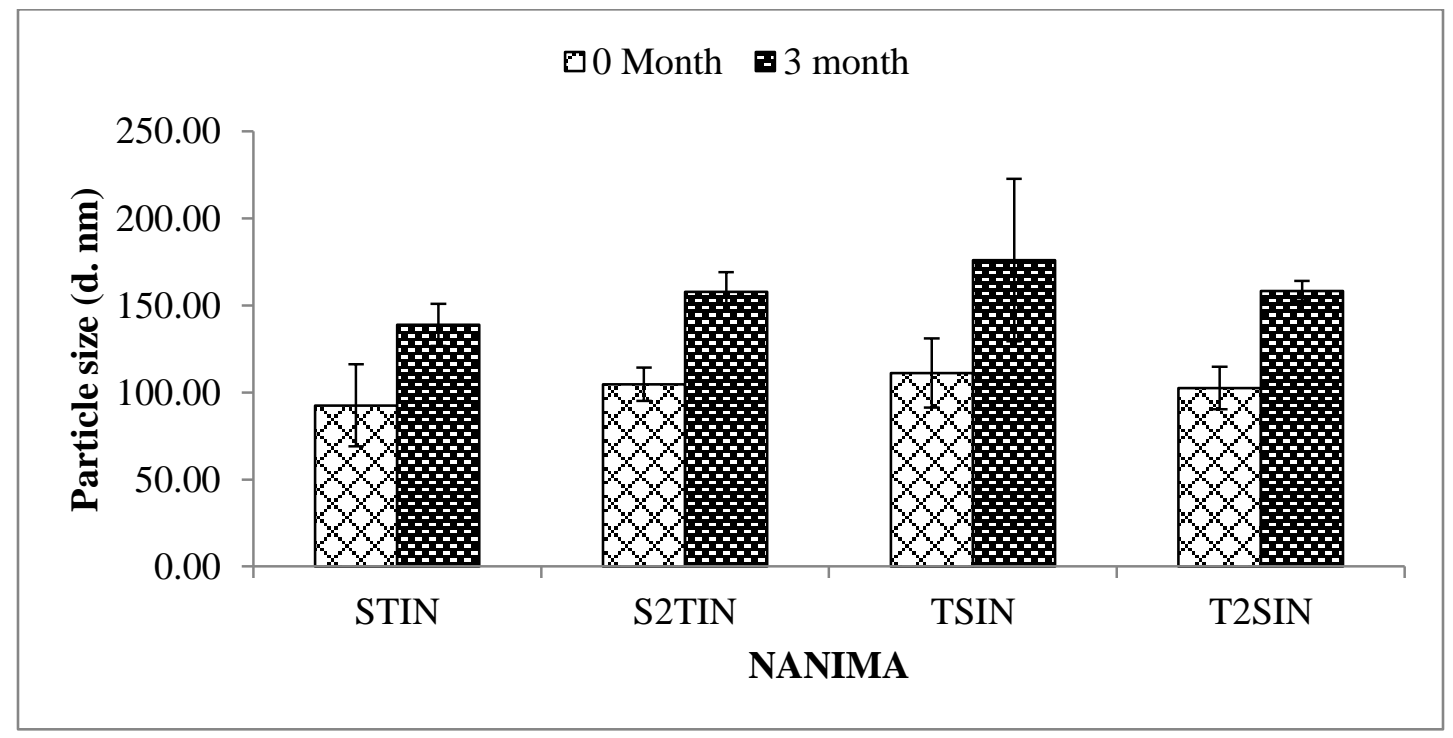

Figure 5: Effect of temperature on particle size of NANIMAs after 3 months of storage. An increase in particle size was observed to be non-significant in all NANIMA formulations.

The disparity of PdI values of all NANIMAs after three months of storage was compared and estimated with respect to zero months. The outcome of effect of temperature on PdI has been shown in Figure 6. The physical stability of nano-formulations may also be explained by the disparity of PdI values. The long term stability of nanosuspensions is favoured by the smaller value of PdI [37]. All NANIMA formulations exhibited increase in PdI values. Among these, STIN (p = 0.065) was found to exhibit the highest and T2SIN $(\mathrm{p}<0.05)$ exhibited the lowest increase in PdI value with respect to zero month. However, S2TIN was found to reveal the lowest value of PdI (zero months) but an increase in PdI was found to be more than T2SIN after three months of storage. Thus, on the basis of this perception, T2SIN may be considered to be the most stable on 
long term stability study followed by TSIN, S2TIN and STIN (i.e., T2SIN > TSIN > S2TIN > STIN). Hence, T80 was found to give better long term stability than SLS to NANIMA formulations.

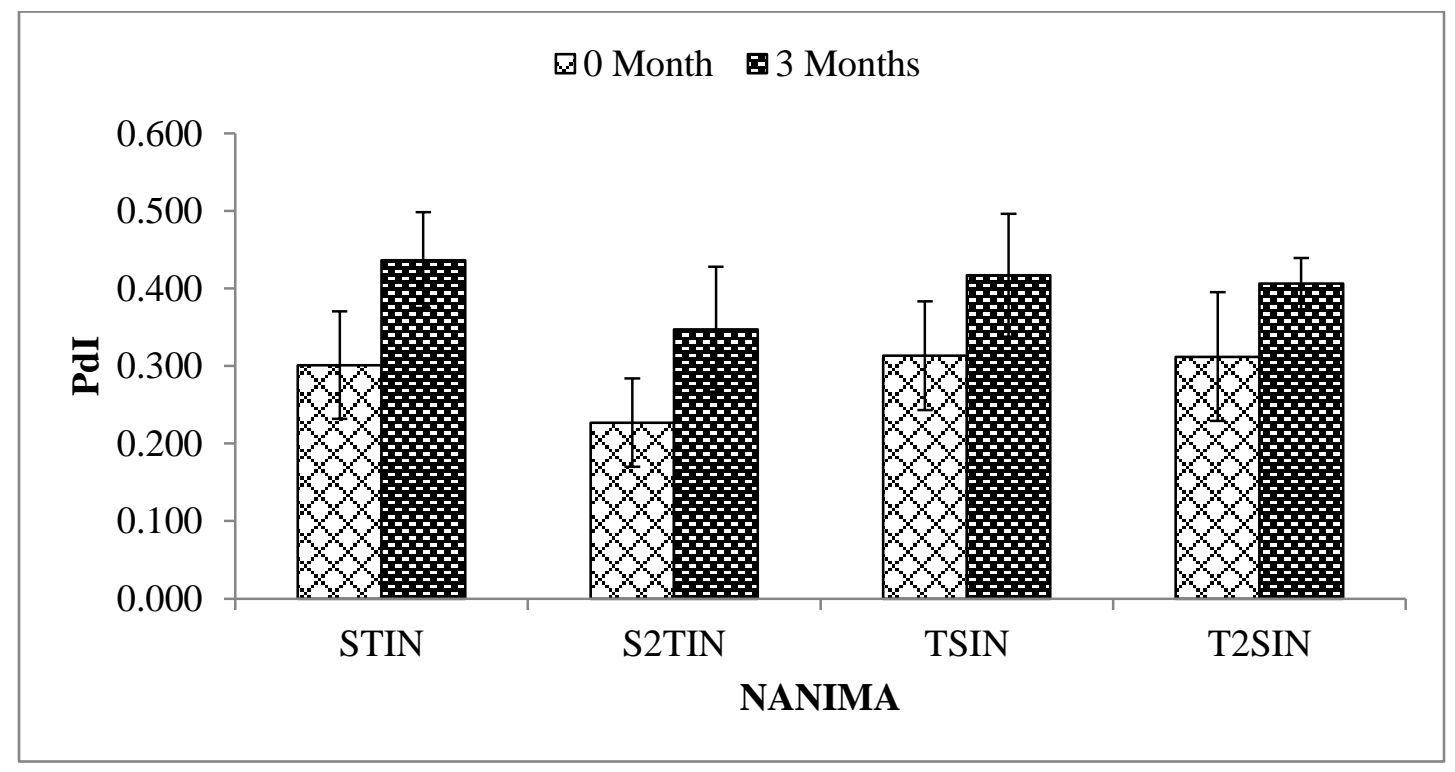

Figure 6: Effect of temperature on polydispersity index (PdI) of NANIMAs after 3 months of storage. STIN exhibited the highest increase $(p=0.065)$ and S2TIN exhibited the lowest increase among all NANIMAs $(p<0.05)$.

The result of influence of temperature on EE has been shown in Figure 7. S2TIN was found to have maximum value of EE while T2SIN exhibited the minimum value among all NANIMA formulations after 3 months. However, the NANIMA formulations were found to have a decreased value of EE (with respect to zero month) when analyzed after the storage period. The rate of decrease in EE value (leakage of IMA) was faster in T2SIN ( $p<0.05)$ followed by TSIN ( $p<$ 0.05), S2TIN ( $p<0.05)$ and STIN $(p>0.05)$. A commendable capability was exhibited by STIN to prevent the leakage of IMA among all NANIMA formulations at room temperature $\left(25^{\circ} \mathrm{C}\right)$. Thus, SLS seems to be more effective than T80 in preventing the leakage of IMA. 


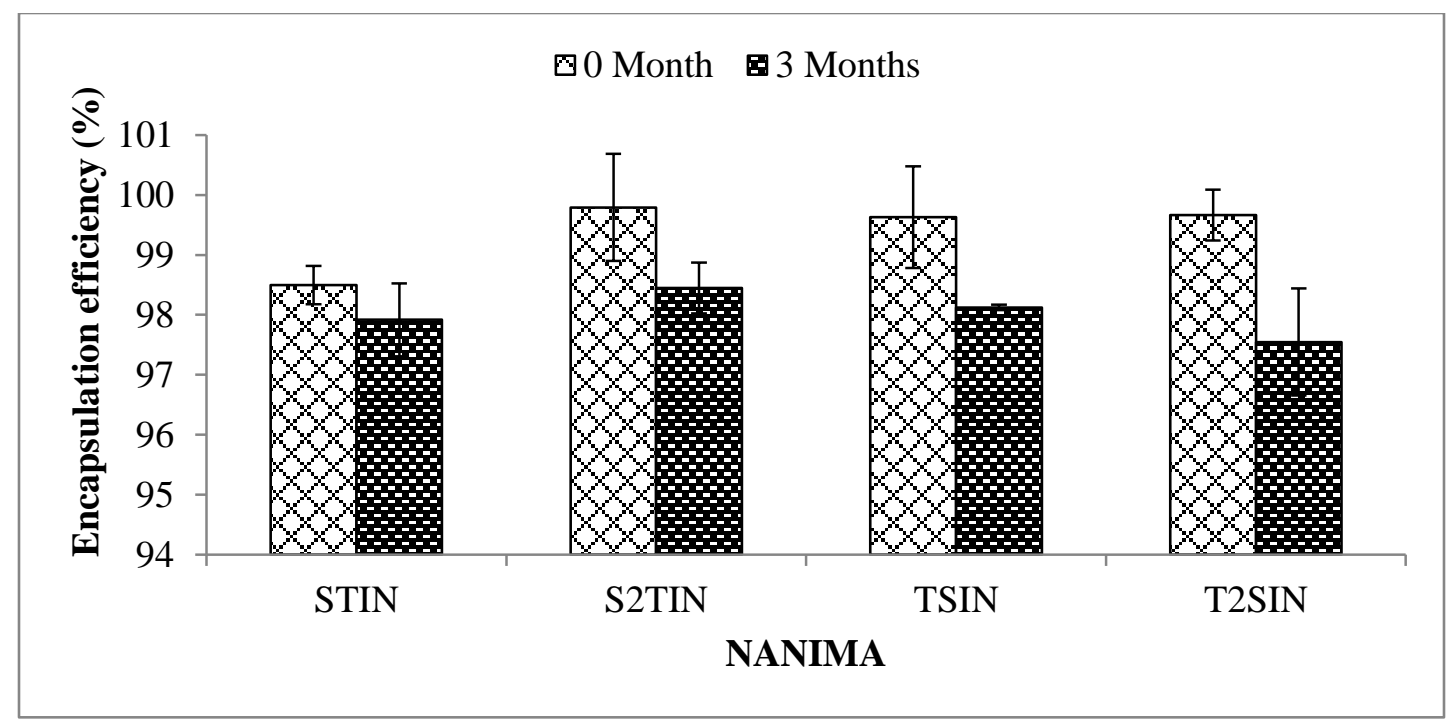

Figure 7: Effect of temperature on encapsulation efficiency (EE \%) of NANIMAs after 3 months of storage. All NANIMAs were assessed to show decrease in EE including T2SIN (p < $0.05)$, TSIN $(p<0.05)$, S2TIN $(p<0.05)$ and STIN $(p>0.05)$.

The effect of temperature on ZP has been shown in Figure 8. The ZP values of all NANIMA formulations were decreased when assessed next to the end of storage period. The newly formulated NLC-dispersions show high value of zeta potential taken after by decrease in values with the increment of passed time. The maximum ZP was estimated for T2SIN followed S2TIN, TSIN and STIN after the end of storage period. However, the rate of reduction of ZP values was faster in case of STIN followed by T2SIN, S2TIN and TSIN (i.e., STIN > T2SIN > S2TIN > TSIN). The reduction in ZP value was found to be non-significant $(\mathrm{p}>0.05)$ for S2TIN, T2SIN and TSIN. Thus, the electrostatic layer was retained everywhere round the NLC particles throughout the system. Consequently, these formulations may be considered to be said to have a great physical stability indeed after the storage of three months. Furthermore, STIN exhibited significant reduction of ZP $(\mathrm{p}<0.05)$. Several factors are responsible for affecting the $\mathrm{ZP}$ values including type and amount surfactants and property of particles to agglomerate over storage. Thus, 
the charges and the dispersion force between particles decreased because of the agglomeration tendency of particles [38].

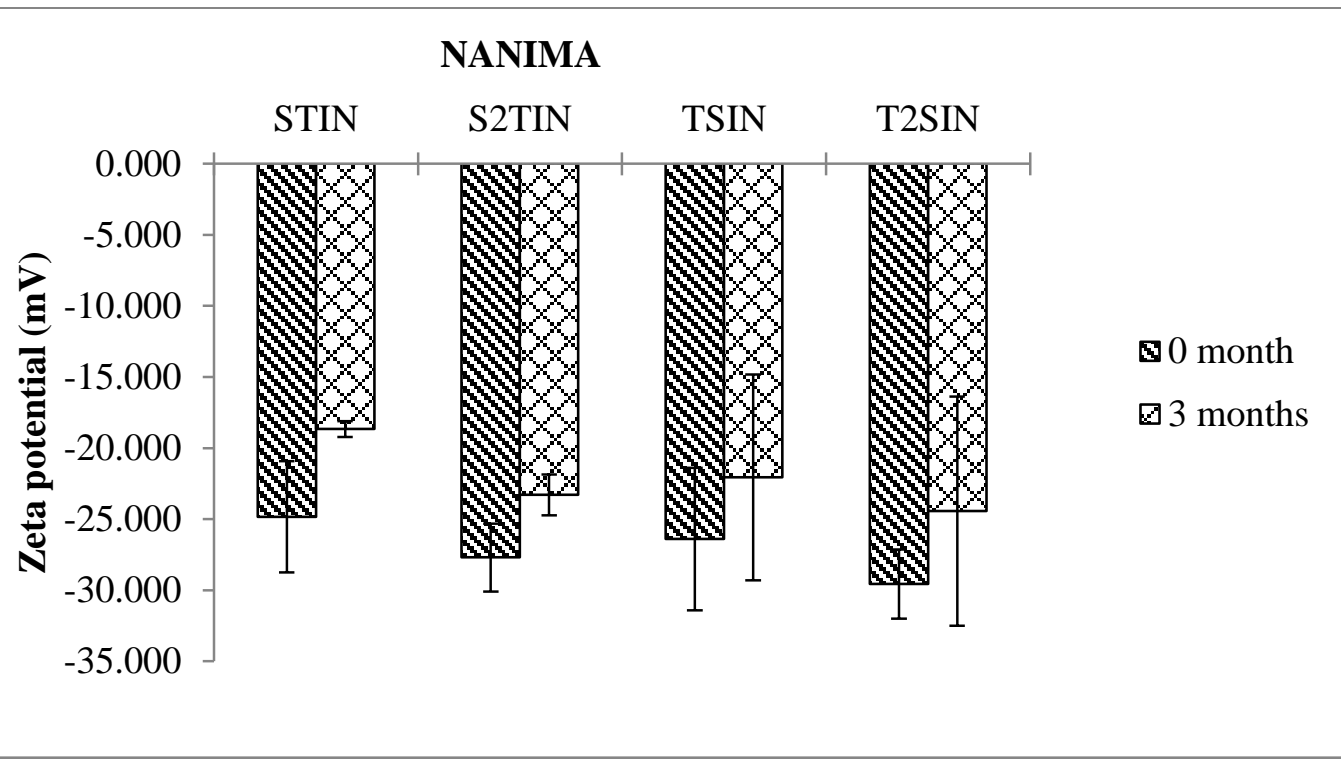

Figure 8: Effect of storage temperature on zeta potential. All NANIMAs were found to exhibit non-significant $(p>0.05)$ decrease of ZP. However, STIN exhibited significant reduction of $\mathrm{ZP}(\mathbf{p}<\mathbf{0 . 0 5})$.

\section{Anti-cancer evaluation}

The cytotoxicity assay (MTT) was done to disclose the anti-cancer prospective of different formulations such as STIN, S2TIN, TSIN, T2SIN, along with IMA alone (Figure 9). The results have shown that S2TIN formulation has significantly higher cytotoxicity, which is found to be superior compared to pure compound IMA. Moreover, the blank exhibited no cytotoxicity within the range used in this study (Table 3).

Table 3: $\mathrm{IC}_{50}$ value of Imatinib (Pure) and its formulations at 72 hours.

\begin{tabular}{|l|c|}
\hline \multicolumn{1}{|c|}{ Formulation } & IC $_{\mathbf{5 0}}(\boldsymbol{\mu M})$ \\
\hline Imatinib (Pure) & $52.5 \pm 3.24$ \\
\hline
\end{tabular}




\begin{tabular}{|l|c|}
\hline STIN & $13.5 \pm 1.0$ \\
\hline S2TIN & $6 \pm 0.2$ \\
\hline TSIN & $24 \pm 1.8$ \\
\hline T2SIN & $31 \pm 1.9$ \\
\hline Blank*** & $\geq 100$ \\
\hline
\end{tabular}

***S2TIN without Imatinib

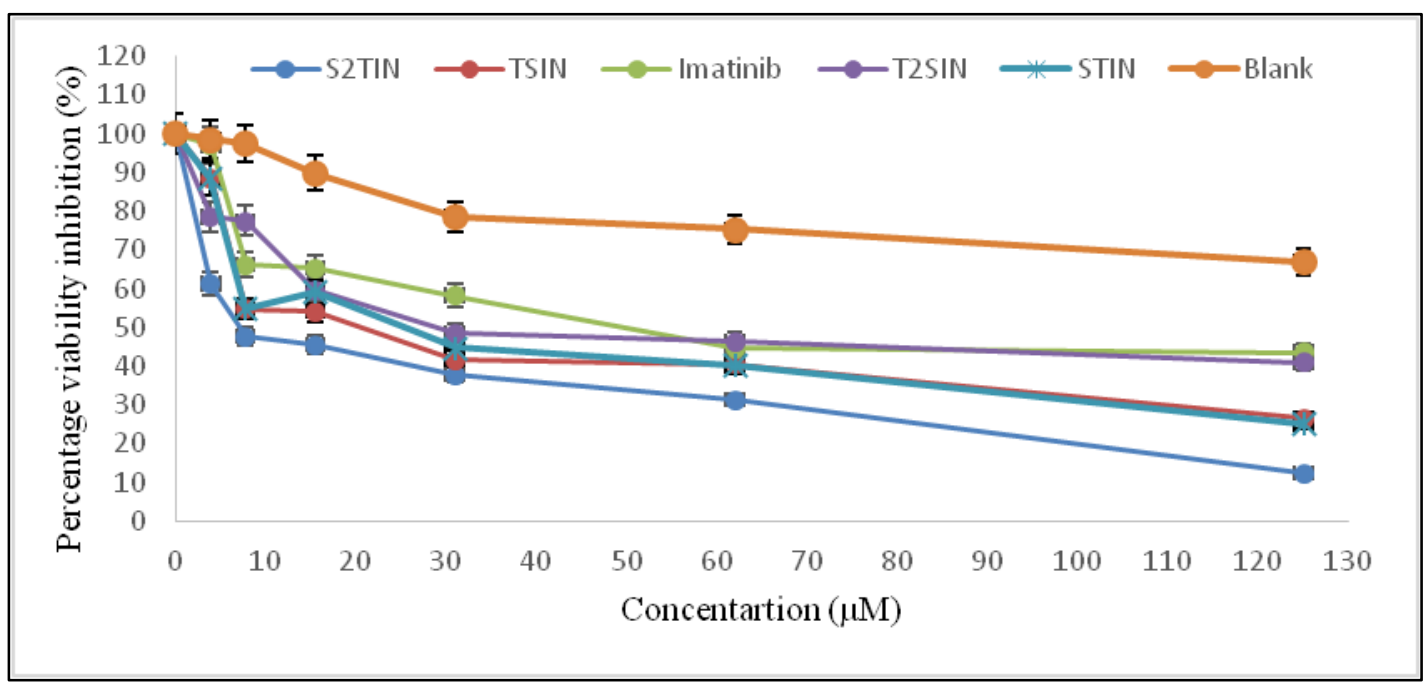

Figure 9: Cytotoxicity of IMA (Pure) and its formulations at 72 hours. Values are represented as average of three replicates.

Furthermore, under the fluorescence microscope, apoptotic, necrotic and viable MCF-7 breast cancer cells were scored. This also included the (untreated control cells; 200 cells were counted randomly and differentially. The assay showed that IMA 52.5 $\pm 3.24 \mu \mathrm{M}$ and S2TIN $6 \pm 0.2 \mu \mathrm{M}$ has an equal number of apoptosis cells displayed. There was a time depended on an increase in the dead cells, as observed at 48 and $72 \mathrm{~h}$ (Figure 10 A-E). Early apoptosis was apparent within the fragmented DNA by intercalated AO. The fluorescent bright-green color could only be found in 
treated cells. In comparison, it was found that untreated cells had a green, intact nuclear structure. Differential scoring of treated cells (200 cell population) as shown in Figure 10 F shows that the difference in positive apoptotic cells was statistically significant for 48 and $72 \mathrm{~h}(\mathrm{p}<0.05)$. On the other hand, as seen in Figure 10 F, there was no statistically significant difference $(\mathrm{p}>0.05)$ among the IMA and S2TIN formulation in various periods during therapy (48 and $72 \mathrm{~h}$ ).

In clinical use, cytotoxic medications have various functional challenges, such as higher toxicity, low cancer cell specificity and susceptibility to pharmaceutical formulations. In the case of IMA, cardiotoxicity is the primary concern [39]. Hence, reducing the effective dose will be an achievement in the treatment strategies. Our results obtained from the cytotoxicity assays revealed that S2TIN formulation has more significant potential compared to the IMA alone. Additionally, we checked the possibility of S2TIN to exert the apoptosis in different doses. The phase of programmed cell death or apoptosis is typically distinguished by distinct morphological features and energy-dependent biochemical pathways [40]. Imatinib has earlier showed to induce apoptosis in breast cancer in vitro [41]. Our studies have revealed that the interacting dye which binds with DNA is visible evidence of induction of early and late apoptosis in the given doses. Moreover, the quantification of cell population in different phases suggested that S2TIN has the potential of inducing apoptosis equivalent to the IMA at lower doses. 

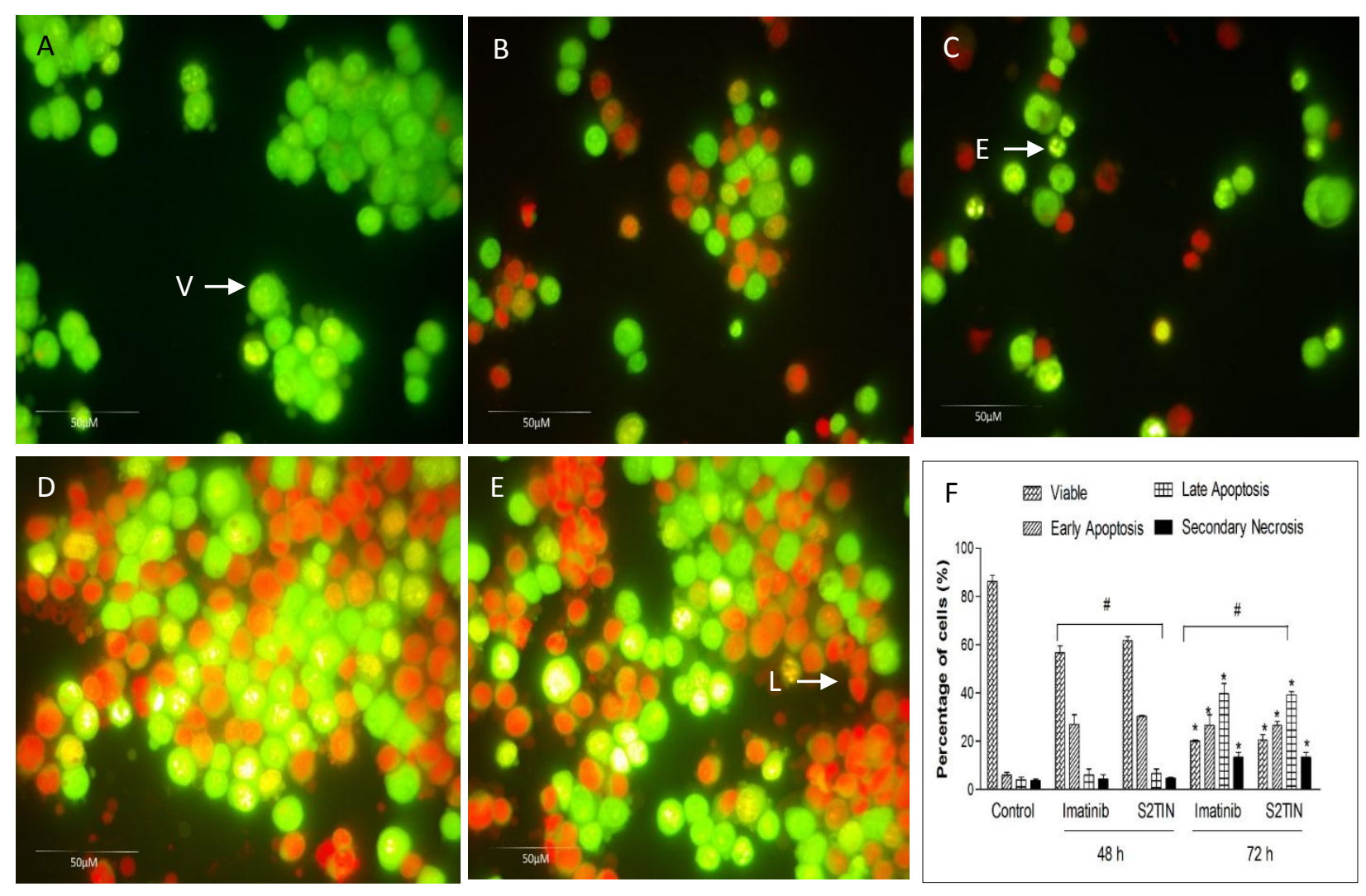

Figure 10: Fluorescent micrographs of acridine orange/propidium iodide double-stained MCF-7 breast cancer cells treated with $\mathrm{IC}_{50}$ concentration of IMA and S2TIN for 48 and $72 \mathrm{~h}$. (A) Untreated cells show normal structure without prominent apoptosis and necrosis (bright green). (B and C) Early apoptosis features seen after $48 \mathrm{~h}$ treatment (bright green) with IMA and S2TIN respectively. (D and E) Late apoptosis was observed at $72 \mathrm{~h}$ (orange colour) with IMA and S2TIN respectively. (F) Percentages of viable, early apoptotic, late apoptosis and secondary necrotic cells after treatment. An asterisk * indicates $\mathrm{p}<0.05$ compared to control, and asterisk \# indicates no significant difference between groups. VI: Viable cells; EA: Early apoptosis; LA: Late apoptosis. Each experiment was performed in triplicate.

\section{Conclusion}

NANIMA (NLC containing Imatinib) formulations were successfully prepared and exhibited commendable outcomes for characterization parameters and evaluation studies. Different 
ingredients including stearic acid, sesame oil and surfactants (SLS and T80) were used in the preparation. On the basis of studied variables, S2TIN may be considered to be the optimum composition among all. The smaller size $(\sim 100 \mathrm{~nm})$ and lipid nature NANIMAs make sure adequate penetration of IMA over different membranous obstacles that possibly will bring about acceptable curing outcomes. Moreover, the release study recommends therapeutic effect for extended period of time on the target site. Furthermore, the high value of EE (>95\%) can be predicted to be an encouraging aspect for improved remedial effectiveness as revealed by cell line studies.

The in vitro anti-cancer potential NANIMAs were evaluated after performing cytotoxicity studies on MCF7 cells. The anti-cancer effect of IMA loaded in NLC was explored to be 8.75 times (S2TIN) superior to IMA alone. Accordingly, the reduced dose will give enhanced anti-cancer effect on cancer patients. Consequently, a lesser amount of adverse effects and exposure of body will be observed. The commendable outcome observed by S2TIN may be credited to the mutual effect of not only of its small size, lipid nature and entrapment efficiency but also the type of ingredients selected, optimized quantities of ingredients and method used. Therefore, the NANIMA formulation of S2TIN composition may be predictably be considered suitable for active cure of cancer after carrying out the studies on animal models.

\section{Funding}

This research was funded by Deanship of Scientific Research, Jazan University, Jazan, KSA, grant number (JUP8/000335).

\section{Acknowledgement}

Authors express gratitude to Research Lab, College of Pharmacy, Jazan University for allowing to carry out the research activities and all necessary facilities were provided.

\section{Author Contributions}


Conceptualization, H.A.M. and A.A.A.; Methodology, M.I.A. and S.M.; Software, M.I.A.; Validation, M.I.A., S.M. and M.A.A; Formal Analysis, H.A.A.; Investigation, M.I.A. and S.M.; Resources, M.H.S.; Data Curation, M.I.A. and M.A.A.; Writing - Original Draft Preparation, M.I.A. and S.M.; Writing - Review \& Editing, H.A.M. and M.A.A; Visualization, R.A.A.; Supervision, A.A.A.; Project Administration, H.A.M.

\section{Institutional Review Board Statement}

Not applicable

\section{Informed Consent Statement}

Not applicable

\section{Data Availability Statement}

Not applicable

\section{Conflict of interest}

The authors declare no conflict of interest.

\section{References}

1. Harbeck, N.; Penault-Llorca, F.; Cortes, J.; et al. Breast cancer. Nat Rev Dis Primers. 2019;5(1):66

2. Chen, Y.; Zhang, H.; Zhang, Y. Targeting receptor tyrosine kinase EphB4 in cancer therapy. Semin Cancer Biol. 2019;56:37-46.

3. Hunter, T. Treatment for chronic myelogenous leukemia: the long road to imatinib. J Clin Invest. 2007;117(8):2036-2043.

4. Vazquez-Martin, A.; Colomer, R.; Brunet, J.; Lupu, R.; Menendez, J.A. Overexpression of fatty acid synthase gene activates HER1/HER2 tyrosine kinase receptors in human breast epithelial cells. Cell Prolif. 2008;41(1):59-85. 
5. Agrawal, A.; Gutteridge, E.; Gee, J.M.; Nicholson, R.I.; Robertson, J.F. Overview of tyrosine kinase inhibitors in clinical breast cancer. Endocrine-related Cancer. 2005;12 Suppl 1:S135-44.

6. Chambers, T.P.; Santiesteban, L.; Gomez, D.; Chambers, J.W. Sab mediates mitochondrial dysfunction involved in imatinib mesylate-induced cardiotoxicity. Toxicology. 2017;382:24-35.

7. Rizwanullah, M.; Ahmad, J.; Amin, S. Nanostructured Lipid Carriers: A Novel Platform for Chemotherapeutics. Curr Drug Deliv. 2016;13(1):4-26.

8. Yingchoncharoen, P.; Kalinowski, D.S.; Richardson, D.R. Lipid-Based Drug Delivery Systems in Cancer Therapy: What Is Available and What Is Yet to Come. Pharmacol Rev. 2016;68(3):701-787.

9. Makeen, H.A.; Mohan, S.; Al-Kasim, M.A, et al. Gefitinib loaded nanostructured lipid carriers: characterization, evaluation and anti-human colon cancer activity in vitro. Drug Deliv. 2020;27(1):622-631.

10. Patlolla, R.R.; Chougule, M.; Patel, A.R.; Jackson, T.; Tata, P.N.; Singh, M. Formulation, characterization and pulmonary deposition of nebulized celecoxib encapsulated nanostructured lipid carriers. J Control Release. 2010;144(2):233-241.

11. Taratula, O.; Kuzmov, A.; Shah, M.; Garbuzenko, O.B.; Minko, T. Nanostructured lipid carriers as multifunctional nanomedicine platform for pulmonary co-delivery of anticancer drugs and siRNA. J Control Release. 2013;171(3):349-357.

12. Alam, M.I.; Baboota, S.; Ahuja, A. et al. Pharmacoscintigraphic evaluation of potential of lipid nanocarriers for nose-to-brain delivery of antidepressant drug. Int $J$ Pharm. 2014;470(1-2):99-106. 
13. Shete, H.K.; Selkar, N.; Vanage, G.R.; Patravale, V.B. Tamoxifen nanostructured lipid carriers: enhanced in vivo antitumor efficacy with reduced adverse drug effects. Int $J$ Pharm. 2014;468(1-2):1-14.

14. Madane, R.G.; Mahajan, H.S. Curcumin-loaded nanostructured lipid carriers (NLCs) for nasal administration: design, characterization, and in vivo study. Drug Deliv. 2016;23(4):1326-1334.

15. Yang, X.Y.; Li, Y.X.; Li, M.; Zhang, L.; Feng, L.X.; Zhang, N. Hyaluronic acid-coated nanostructured lipid carriers for targeting paclitaxel to cancer. Cancer Lett. 2013;334(2):338-345.

16. Alam, M.I.; Baboota, S.; Ahuja, A.; Ali, M.; Ali, J.; Sahni, J.K. Intranasal infusion of nanostructured lipid carriers (NLC) containing CNS acting drug and estimation in brain and blood. Drug Deliv. 2013;20(6):247-51.

17. Alam, M.I.; Baboota, S.; Ahuja, A.; Ali, M.; Ali, J.; Sahni, J.K. Intranasal administration of nanostructured lipid carriers containing CNS acting drug: pharmacodynamic studies and estimation in blood and brain. J Psychiatr Res. 2012;46(9):1133-1138.

18. Saedi, A.; Rostamizadeh, K.; Parsa, M.; Dalali, N.; Ahmadi, N. Preparation and characterization of nanostructured lipid carriers as drug delivery system: Influence of liquid lipid types on loading and cytotoxicity. Chem Phys Lipids. 2018;216:65-72.

19. Syam, S.; Abdelwahab, S.I.; Al-Mamary, M.A.; Mohan, S. Synthesis of chalcones with anticancer activities. Molecules. 2012;17(6):6179-6195.

20. Mozafari, M.R.; Pardakhty, A.; Azarmi, S.; Jazayeri, J.A.; Nokhodchi, A.; Omri, A. Role of nanocarrier systems in cancer nanotherapy. J Liposome Res. 2009;19(4):310-321. 
21. Danaei, M.; Dehghankhold, M.; Ataei, S. et al. Impact of Particle Size and Polydispersity Index on the Clinical Applications of Lipidic Nanocarrier Systems. Pharmaceutics. 2018; $10(2): 57$.

22. Eggersdorfer, M.L.; Pratsinis, S.E. The Structure of Agglomerates consisting of Polydisperse Particles. Aerosol Sci Technol. 2012;46(3):347-353.

23. Şenel, B.; Öztürk, A.A. New approaches to tumor therapy with siRNA-decorated and chitosan-modified PLGA nanoparticles. Drug Dev Ind Pharm. 2019;45(11):1835-1848.

24. Gonzalez-Mira, E.; Egea, M.A.; Souto, E.B.; Calpena, A.C.; García, M.L. Optimizing flurbiprofen-loaded NLC by central composite factorial design for ocular delivery. Nanotechnology. 2011;22(4):045101.

25. Sanad, R.A.; Abdelmalak, N.S.; Elbayoomy, T.S.; Badawi, A.A. Formulation of a novel oxybenzone-loaded nanostructured lipid carriers (NLCs). AAPS PharmSciTech. 2010; $11(4): 1684-1694$.

26. How, C.W.; Rasedee, A.; Abbasalipourkabir, R. Characterization and cytotoxicity of nanostructured lipid carriers formulated with olive oil, hydrogenated palm oil, and polysorbate 80. IEEE Trans Nanobioscience. 2013;12(2):72-78.

27. Westesen, K.; Bunjes, H.; Koch, M.H.J. Physicochemical characterization of lipid nanoparticles and evaluation of their drug loading capacity and sustained release potential. J Control Rel. 1997;48(2-3), 223-236.

28. Aslam, M.; Aqil, M.; Ahad, A.; Najmi, A.K.; Sultana, Y.; Ali, A. Application of BoxBehnken design for preparation of glibenclamide loaded lipid based nanoparticles: Optimization, in vitro skin permeation, drug release and in vivo pharmacokinetic study. $J$ Molecular Liquids. 2016;219, 897-908, 
29. Intakhab, A.M.; Baboota, S.; Ahuja, A.; Ali, M.; Ali, J.; Kaur, S.J. Nanostructured lipid carrier containing CNS acting drug: formulation, optimization and evaluation. Current Nanosci. 2011;7(6):1014-27.

30. Jain, K.; Sood, S.; Gowthamarajan, K. Optimization of artemether-loaded NLC for intranasal delivery using central composite design. Drug Deliv. 2015;22(7):940-954.

31. Thang, L.Q.; Hanh, N.; Duong, D. Study on cause-effect relations and optimization of exemestane-loaded nanostructured lipid carriers. Int J Pharm Pharm Sci. 2017;9(5):68-74.

32. Khosa, A.; Reddi, S.; Saha, R.N. Nanostructured lipid carriers for site-specific drug delivery. Biomed Pharmacother. 2018;103:598-613.

33. Zhang, W.; Li, X.; Ye, T. et al. Design, characterization, and in vitro cellular inhibition and uptake of optimized genistein-loaded NLC for the prevention of posterior capsular opacification using response surface methodology. Int J Pharm. 2013;454(1):354-366.

34. Roohinejad, S.; Greiner, R.; Oey, I. and Wen, J. eds., 2018. Emulsion-based systems for delivery of food active compounds: formation, application, health and safety. First Edition. John Wiley \& Sons Ltd.

35. Kaur, P.; Garg, T.; Rath, G.; Murthy, R.S. Goyal, A.K. Development, optimization and evaluation of surfactant-based pulmonary nanolipid carrier system of paclitaxel for the management of drug resistance lung cancer using Box-Behnken design. Drug Deliv. 2016;23(6):1912-1925.

36. Elmowafy, M.; Ibrahim, H.M.; Ahmed, M.A.; Shalaby, K.; Salama, A.; Hefesha, H. Atorvastatin-loaded nanostructured lipid carriers (NLCs): strategy to overcome oral delivery drawbacks. Drug Deliv. 2017;24(1):932-941.

37. Lakshmi, P.; Kumar, G.A. Nanosuspension technology: A review. Int J Pharm Sci. 2010;2(4):35-40. 
38. Nakatuka, Y.; Yoshida, H.; Fukui, K.; Matuzawa, M. The effect of particle size distribution on effective zeta-potential by use of the sedimentation method. Advanced Powder Technology. 2015;26(2):650-6.

39. Kerkelä, R.; Grazette, L.; Yacobi, R. et al. Cardiotoxicity of the cancer therapeutic agent imatinib mesylate. Nat Med. 2006;12(8):908-916.

40. Elmore, S. Apoptosis: a review of programmed cell death. Toxicol Pathol. 2007;35(4):495516.

41. Weigel, M.T.; Dahmke, L.; Schem, C. et al. In vitro effects of imatinib mesylate on radiosensitivity and chemosensitivity of breast cancer cells. BMC Cancer. 2010;10:412. 